\title{
Effects of different anesthetic methods on immune function and oxidative stress in patients undergoing laparoscopic herniorrhaphy
}

Lei Wu, Jingying Liu, Yue Chen, Lei Tan, Qiannan Fan, Mingjie Zhong, Hao Wu

Department of Anesthesiology, The First People's Hospital of Suqian, Suqian, China

Videosurgery Miniinv 2021; 16 (2): 329-335

DOI: https://doi.org/10.5114/wiitm.2021.105528

\begin{abstract}
Introduction: The influence of anesthetic method on the immune function and oxidative stress in patients receiving laparoscopic herniorrhaphy remains largely unknown.

Aim: To assess the effects of different anesthetic methods on the immune function and oxidative stress in patients undergoing laparoscopic herniorrhaphy.

Material and methods: A total of 280 eligible patients were randomly divided into two groups to receive total intravenous anesthesia (TIVA group) and inhalation anesthesia (IA group). T lymphocyte subsets, oxidative stress response indices and inflammatory factor levels were measured before anesthesia, at the end of the operation, and 1 and 3 days after the operation. The incidence rates of adverse reactions were compared.

Results: At the end of and 1 day after the operation, $C D 3^{+}$and $C D 4^{+} T$ cells and $C D 4^{+} / C D 8^{+}$decreased in both groups compared with those before anesthesia $(p<0.05)$. Three days after the operation, $C D 3^{+}$and $C D 4^{+} T$ cells decreased in both groups compared with those before anesthesia, which were higher in the TIVA group $(p<0.05)$. Interleukin-6 and C-reactive protein levels were significantly lower in the TIVA group $(p<0.05)$. At the end of and 1 and 3 days after the operation, oxidative stress response indices superoxide dismutase, catalase and glutathione peroxidase declined in both groups compared with those before anesthesia, which were higher in the IA group $(p<0.05)$. The incidence rate of adverse reactions was significantly higher in the IA group $(p=0.002)$.

Conclusions: Compared with IA, TIVA has less effect on the immune function and oxidative stress of patients undergoing laparoscopic herniorrhaphy, and controls the inflammatory response more effectively, accompanied by a lower incidence rate of adverse reactions.
\end{abstract}

Key words: total intravenous anesthesia, laparoscopic herniorrhaphy, immune function, oxidative stress.

\section{Introduction}

Inguinal hernia refers to the protrusion of the abdominal cavity contents to the body surface through a defect of the abdominal wall in the inguinal region, which can be cured by standard surgical therapy, and inguinal hernia in adults should be treated surgically. According to the literature [1, 2], the incidence rate of inguinal hernia is about $0.1-0.5 \%$. Cur- rently, laparoscopic herniorrhaphy is a commonly adopted method of herniorrhaphy in clinical practice, which has the advantages of less trauma, a clear field of vision, high safety and reliability in tissue separation and mesh placement, and remarkable efficacy. However, all surgery leads to trauma and will cause a stress response, inducing varying degrees of damage to the immune function $[3,4]$. Different an- 
esthetic methods lead to different stress response intensities and immune function damage $[5,6]$. At present, the influences of different anesthetic methods on the immune function and oxidative stress in patients are studied mostly in tumorectomy, whereas such influences in laparoscopic herniorrhaphy are relatively less investigated. Herniorrhaphy is more common in clinical practice.

\section{Aim}

Therefore, we herein evaluated the influences of different anesthetic methods on the immune function and oxidative stress in patients undergoing laparoscopic herniorrhaphy, aiming to find out a more suitable anesthetic method for maximizing the efficacy of surgical therapy.

\section{Material and methods}

\section{General information}

A total of 280 patients undergoing laparoscopic herniorrhaphy in our hospital between January 2018 and November 2020 were prospectively selected (Figure 1). Inclusion criteria: (1) Patients meeting the diagnostic criteria in Guidelines for diagnosis and treatment on the adult groin hernia (2018 edition) [7], and (2) those definitely diagnosed with direct or indirect inguinal hernia based on medical history in- quiry and auxiliary examinations. Exclusion criteria: (1) Patients with contraindications for total intravenous anesthesia (TIVA) or inhalation anesthesia (IA), (2) those with uncorrected coagulation dysfunction, (3) underage patients, (4) those with recurrent hernia, (5) those with femoral hernia, or (6) those with other surgery-intolerable contraindications. The 280 patients were divided into the TIVA group $(n=140)$ and the IA group $(n=140)$ using the random number table method. In the TIVA group, there were 76 males and 64 females, aged $39-71$ years old, with a mean of $59.2 \pm 6.3$ years old. In the IA group, there were 72 males and 68 females, aged 41-73 years old, with a mean of $59.4 \pm 5.9$ years old. No significant differences were observed in the clinical baseline data between the two groups $(p>0.05)$ (Table I). The present study was approved by the Ethics Committee of our hospital, and all patients and their family members signed the informed consent.

\section{Anesthesia methods}

At 30 min before the operation, patients in both groups were intramuscularly injected with $0.5 \mathrm{mg}$ of atropine (NMPN: H32020166, Jiangsu Lianshui Pharmaceutical Co., Ltd.) and $5 \mathrm{mg}$ of diazepam (NMPN: H41020631, Tianjin Pharma Jiaozuo Co., Ltd.). Anesthesia was induced by in-

Patients undergoing laparoscopic hernia repair $(n=290)$

With contraindications of total intravenous anesthesia $(n=2)$

With contraindications of inhalation anesthesia $(n=1)$

With uncorrected coagulation dysfunction $(n=2)$

Age $<18(n=2)$

With recurrent hernia $(n=1)$

With femoral hernia $(n=2)$

Patients included in this study $(n=280)$

Figure 1. Study enrollment chart 
Table I. Baseline clinical data

\begin{tabular}{|lcccc|}
\hline Parameter & TIVA group $(n=140)$ & IA group $(n=140)$ & $\chi^{2}, t$ & $P$-value \\
\hline Gender: & & & $\chi^{2}=0.229$ & 0.632 \\
\hline Male & 76 & 68 & & \\
\hline Female & 64 & $59.4 \pm 5.9$ & $t=0.274$ & 0.784 \\
\hline Age [years] & $59.2 \pm 6.3$ & $58.8 \pm 6.5$ & $t=1.327$ & 0.185 \\
\hline Body weight [kg] & $57.8 \pm 6.1$ & 70 & $\chi^{2}=1.023$ & 0.600 \\
\hline Direct hernia & 78 & 62 & & \\
\hline Indirect hernia & 56 & 8 & &
\end{tabular}

travenous injection of $4 \mu \mathrm{g} / \mathrm{kg}$ fentanyl (NMPN: H42022076, Yichang Humanwell Pharmaceutical Co., Ltd.) and $0.1 \mathrm{mg} / \mathrm{kg}$ midazolam, followed by $2 \mathrm{mg} / \mathrm{kg}$ propofol (NMPN: H20010368, Xi'an Libang Pharmaceutical Co., Ltd.). When the patients lost consciousness, they were intravenously injected with $0.15 \mathrm{mg} / \mathrm{kg}$ cisatracurium besilate (NMPN: H20060869, Shanghai Hengrui Pharmaceutical Co., Ltd.). After the patients' muscles were relaxed, endotracheal intubation was conducted. During the operation, cisatracurium besilate was intravenously injected every $30 \mathrm{~min}$ (Dosage: $10 \mathrm{mg}$ of drug was dissolved in $5 \mathrm{ml}$ of sterile water for injection. The dose was adjusted according to the patients' neuromuscular function) to maintain muscular relaxation, and then mechanical ventilation was carried out. Anesthesia was induced in both groups using the same method. In the IA group, anesthesia was maintained by inhalation of isoflurane (NMPN: X19990127, Abbott Laboratories Limited) with end-tidal concentration of $1 \%$, which was stopped 30 min before the end of the operation. In the TIVA group, anesthesia was maintained by continuous intravenous infusion of remifentanil (NMPN: H42022076, Yichang Humanwell Pharmaceutical Co., Ltd.) and propofol (NMPN: H20010368, Xi'an Libang Pharmaceutical Co., Ltd.) using a micro-pump. Propofol was maintained at a dose of $4 \mathrm{mg} /(\mathrm{kg} \cdot \mathrm{h})$ until $10 \mathrm{~min}$ before the end of the operation, and remifentanil was given at $0.1 \mu \mathrm{g} /(\mathrm{kg} \cdot \min )$ until $5 \mathrm{~min}$ before the end of the operation. In this study, the operation was performed by members of the same operation team, and the anesthesia depth monitor (G9L multi-parameter anesthesia depth monitor, Danmeter
ApS, Denmark) was used to monitor anesthesia throughout the operation.

\section{Observation of anesthetic effects}

The operation time, anesthesia duration and extubation time were recorded in both groups.

\section{Measurement of immune cell levels}

Before anesthesia, at the end of the operation, and during 7:00-8:00 am at 1 and 3 days after the operation, $5 \mathrm{ml}$ of venous blood was collected from each patient, and the immune-related indices, including $\mathrm{T}$ lymphocyte subsets cluster of differentiation $3^{+}\left(\mathrm{CD}^{+}\right), \mathrm{CD}^{+}$and $\mathrm{CD}^{+}$, and the level of $\mathrm{CD}^{+} /$ $\mathrm{CD}^{+}$were detected in both groups using a Coulter Epics XL flow cytometry (USA).

\section{Measurement of levels of oxidative stress response indices}

Before anesthesia, at the end of the operation, and during 7:00-8:00 am at 1 and 3 days after the operation, $5 \mathrm{ml}$ of venous blood was collected from each patient, and the levels of catalase (CAT), superoxide dismutase (SOD) and glutathione peroxidase (GSH-Px) were measured by the thiobarbituric acid method.

\section{Measurement of levels of inflammatory factors}

Before anesthesia, at the end of the operation, and during 7:00-8:00 am at 1 and 3 days after the operation, $5 \mathrm{ml}$ of venous blood was collected from each patient, and the levels of immune factors were measured. Interleukin-6 (IL-6) and tumor necrosis factor- $\alpha$ (TNF- $\alpha$ ) were determined by enzyme-linked 
immunosorbent assay (ELISA) according to the instructions of the ELISA kits (Shanghai Jining Shiye Co., Ltd.), and the level of C-reactive protein (CRP) was determined by immunoturbidimetry.

\section{Observation of adverse reactions}

The incidence of adverse reactions, including irritability, nausea and excessive sedation, was compared between the two groups.

\section{Statistical analysis}

SPSS 21.0 software was used for statistical analysis. The measurement data in line with normal distribution were expressed as mean \pm standard deviation $(\chi \pm s)$, and the $t$-test was used for comparison between groups. The numerical data were expressed as percentage, and the $\chi^{2}$ test was employed for comparison between groups. $P<0.05$ suggested that a difference was statistically significant.

\section{Results}

\section{Anesthetic effects}

There were no significant differences in the operation time, anesthesia duration and extubation time between the two groups $(p>0.05)$ (Table II).

\section{Levels of immune cells}

At the end of the operation and at 1 day after the operation, $\mathrm{CD}^{+}$and $\mathrm{CD}^{+} \mathrm{T}$ cells and $\mathrm{CD} 4^{+} / \mathrm{CD}^{+}$ were decreased in both groups in contrast with those before anesthesia $(p<0.05)$. At 3 days after the operation, levels of $\mathrm{CD}^{+}$and $\mathrm{CD}^{+} \mathrm{T}$ cells were also lower than those before anesthesia ( $p<0.05)$, and they were significantly higher in the TIVA group than those in the IA group $(p<0.05)$. However, there was no significant difference in the level of $\mathrm{CD}^{+}$ $T$ cells between the two groups at each detection time $(p>0.05)$ (Table III).

\section{Levels of oxidative stress response indices}

At the end of the operation and 1 and 3 days after the operation, the oxidative stress indices SOD, CAT and GSH-Px declined in both groups in comparison with those before anesthesia $(p<0.05)$, and they were significantly higher in the IA group than those in the TIVA group at each time point $(p<0.05)$ (Table IV).

\section{Levels of inflammatory factors}

At the end of the operation and at 1 and 3 days after the operation, there was no significant difference in the level of TNF- $\alpha$ between the two groups

Table II. Anesthetic effects

\begin{tabular}{|lcccc|}
\hline Group & TIVA group $(n=140)$ & IA group $(n=140)$ & $t$ & $P$-value \\
\hline Operation time [min] & $64.15 \pm 2.43$ & $64.38 \pm 2.19$ & 0.832 & 0.406 \\
\hline Anesthesia duration [min] & $68.55 \pm 1.32$ & $68.71 \pm 1.42$ & 0.976 & 0.33 \\
\hline Extubation time [min] & $82.21 \pm 2.45$ & $82.45 \pm 2.53$ & 0.806 & 0.421 \\
\hline
\end{tabular}

Table III. Levels of immune cells at each time point

\begin{tabular}{|lcccccc|}
\hline Index & Group & $N$ & Before anesthesia & At end of operation & 1 day after operation & $\begin{array}{c}3 \text { days after } \\
\text { operation }\end{array}$ \\
\hline CD3+ & TIVA & 140 & $58.55 \pm 5.46(51-63)$ & $52.13 \pm 4.89^{\#}(47-56)$ & $54.41 \pm 4.76^{\#}(50-58)$ & $60.21 \pm 5.68^{\#}(54-67)$ \\
\cline { 2 - 7 } & IA & 140 & $58.89 \pm 5.51(53-64)$ & $48.87 \pm 4.92^{\#}(44-53)$ & $52.56 \pm 4.82^{\#}(48-57)$ & $54.29 \pm 5.74^{*}, \#(51-60)$ \\
\hline CD4+ & TIVA & 140 & $32.36 \pm 3.23(29-36)$ & $26.57 \pm 2.45^{\#}(24-30)$ & $28.73 \pm 3.24^{\#}(27-32)$ & $35.11 \pm 3.45^{\#}(32-38)$ \\
\cline { 2 - 7 } & IA & 140 & $32.86 \pm 3.42(29-36)$ & $25.13 \pm 2.51^{\#}(23-28)$ & $27.66 \pm 3.19^{\#}(24-31)$ & $28.82 \pm 3.28^{*, \#(26-32)}$ \\
\hline CD8+ & TIVA & 140 & $25.68 \pm 2.45(23-28)$ & $24.79 \pm 2.52(22-26)$ & $24.89 \pm 2.62(23-27)$ & $24.22 \pm 2.46(23-27)$ \\
\cline { 2 - 7 } & IA & 140 & $25.76 \pm 2.43(23-28)$ & $24.61 \pm 2.37(23-28)$ & $25.19 \pm 2.43(23-28)$ & $24.78 \pm 2.56(22-28)$ \\
\hline $\begin{array}{l}\text { CD4+/ } \\
\text { CD8+ }\end{array}$ & TIVA & 140 & $1.32 \pm 0.18(1.11-1.52)$ & $1.06 \pm 0.09^{\#}(0.92-1.15)$ & $1.13 \pm 0.11^{\#}(0.90-1.24)$ & $1.32 \pm 0.13(1.16-1.45)$ \\
\cline { 2 - 7 } & IA & 140 & $1.30 \pm 0.20(1.08-1.48)$ & $0.98 \pm 0.08^{\#}(0.89-1.06)$ & $1.08 \pm 0.12^{\#}(0.95-1.17)$ & $1.24 \pm 0.11(1.12-1.32)$ \\
\hline
\end{tabular}

${ }^{*} P<0.05$ vs. TIVA group, ${ }^{*} p<0.05$ vs. before anesthesia. 
( $p>0.05)$. However, the levels of IL-6 and CRP were significantly elevated in contrast with those before anesthesia $(p<0.05)$. Moreover, they were lower in the TIVA group than those in the IA group at the end of the operation and at 1 and 3 days after the operation $(p<0.05)$ (Table V).

\section{Adverse reactions}

The incidence rate of adverse reactions was significantly higher in the IA group than that in the TIVA

Table IV. Levels of oxidative stress response indices group $(25.00 \%$ vs. $11.43 \%)\left(\chi^{2}=9.447, p=0.002\right)$ (Table VI). Probably, IA easily stimulated the respiratory tract, so the incidence rates of irritability and nausea were high.

\section{Discussion}

Surgical trauma brings continuous and varying stimulation to the body, and the acceptance of the traumatic stress response varies in patients [8]. After trauma, the oxidative stress response will be

\begin{tabular}{|lcccccc|}
\hline Index & Group & $N$ & Before anesthesia & At end of operation & 1 day after operation & 3 days after operation \\
\hline SOD & TIVA & 140 & $107.26 \pm 11.27$ & $78.76 \pm 5.27^{\#}$ & $86.42 \pm 5.11^{\#}$ & $98.22 \pm 5.74^{\#}$ \\
{$[\mathrm{U} / \mathrm{ml}]$} & & & $(89.25-120.18)$ & $(73.24-84.13)$ & $(82.15-91.24)$ & $(94.37-103.22)$ \\
\cline { 2 - 7 } & IA & 140 & $106.92 \pm 11.32$ & $60.28 \pm 5.33^{*, \#}$ & $72.38 \pm 4.81^{*, \#}$ & $85.39 \pm 5.25^{*, \#}$ \\
& & & $(94.27-118.24)$ & $(58.23-64.92)$ & $(68.25-76.29)$ & $(84.27-90.17)$ \\
\hline CAT & TIVA & 140 & $80.34 \pm 7.21$ & $71.56 \pm 4.41^{\#}$ & $74.73 \pm 3.22^{\#}$ & $77.12 \pm 3.45^{\#}$ \\
{$[\mathrm{U} / \mathrm{ml}]$} & & & $(73.22-87.73)$ & $(67.25-75.49)$ & $(71.22-77.43)$ & $(74.26-80.18)$ \\
\cline { 2 - 7 } & IA & 140 & $80.45 \pm 7.33$ & $63.14 \pm 4.51^{*, \#}$ & $65.62 \pm 3.14^{*, \#}$ & $69.82 \pm 3.22^{*, \#}$ \\
& & & $(73.25-87.19)$ & $(59.25-67.288)$ & $(64.29-68.56)$ & $(66.25-73.41)$ \\
\hline GSH-Px & TIVA & 140 & $196.34 \pm 15.43$ & $154.72 \pm 12.45^{\#}$ & $174.82 \pm 12.78^{\#}$ & $182.21 \pm 12.45^{\#}$ \\
{$[\mathrm{U} / \mathrm{ml}]$} & & & $(174.23-215.19)$ & $(135.27-167.25)$ & $(159.28-186.45)$ & $(175.43-193.24)$ \\
\cline { 2 - 7 } & IA & 140 & $197.04 \pm 16.38$ & $134.62 \pm 12.43^{*, \#}$ & $155.11 \pm 12.49^{*, \#}$ & $164.71 \pm 12.52^{*, \#}$ \\
& & & $(184.23-214.38)$ & $(123.29-148.24)$ & $(143.27-167.32)$ & $(158.28-178.34)$ \\
\hline
\end{tabular}

${ }^{\star} P<0.05$ vs. group $A,{ }^{\#} P<0.05$ vs. before anesthesia.

Table V. Levels of inflammatory factors

\begin{tabular}{|c|c|c|c|c|c|c|}
\hline Index & Group & $N$ & Before anesthesia & At end of operation & 1 day after operation & 3 days after operation \\
\hline \multirow[t]{2}{*}{$\begin{array}{l}\text { IL-6 } \\
{[\mathrm{pg} / \mathrm{ml}]}\end{array}$} & TIVA & 140 & $\begin{array}{c}58.97 \pm 5.46 \\
(54.38-63.27) \\
\end{array}$ & $\begin{array}{l}66.89 \pm 5.22^{\#} \\
(61.27-72.34) \\
\end{array}$ & $\begin{array}{c}64.39 \pm 4.12^{\#} \\
(60.28-68.29)\end{array}$ & $\begin{array}{c}60.21 \pm 3.56^{\#} \\
(57.28-63.42) \\
\end{array}$ \\
\hline & IA & 140 & $\begin{array}{c}58.29 \pm 5.31 \\
(53.29-64.02)\end{array}$ & $\begin{array}{l}79.22 \pm 5.32^{*, \#} \\
(74.25-84.32)\end{array}$ & $\begin{array}{l}69.02 \pm 4.23^{\star, \#} \\
(65.29-73.22)\end{array}$ & $\begin{array}{l}64.32 \pm 3.54^{\star, \#} \\
(62.45-67.92)\end{array}$ \\
\hline \multirow[t]{2}{*}{$\begin{array}{l}\text { TNF- } \alpha \\
{[\mathrm{ng} / \mathrm{ml}]}\end{array}$} & TIVA & 140 & $\begin{array}{c}33.31 \pm 2.22 \\
(30.22-35.46)\end{array}$ & $\begin{array}{c}31.52 \pm 2.35 \\
(30.34-33.42)\end{array}$ & $\begin{array}{c}31.54 \pm 2.32 \\
(29.87-33.46)\end{array}$ & $\begin{array}{c}29.97 \pm 2.41 \\
(28.98-33.15)\end{array}$ \\
\hline & IA & 140 & $\begin{array}{c}33.42 \pm 2.32 \\
(29.32-35.42)\end{array}$ & $\begin{array}{c}32.16 \pm 2.44 \\
(29.48-34.87)\end{array}$ & $\begin{array}{c}31.61 \pm 2.24 \\
(29.68-33.57)\end{array}$ & $\begin{array}{c}29.81 \pm 2.33 \\
(28.76-32.41)\end{array}$ \\
\hline \multirow[t]{2}{*}{$\begin{array}{l}\text { CRP } \\
{[\mu \mathrm{g} / \mathrm{ml}]}\end{array}$} & TIVA & 140 & $\begin{array}{c}71.31 \pm 5.41 \\
(67.89-76.34)\end{array}$ & $\begin{array}{c}80.43 \pm 4.89^{\#} \\
(76.43-84.38)\end{array}$ & $\begin{array}{c}75.84 \pm 4.71^{\#} \\
(71.46-78.94)\end{array}$ & $\begin{array}{c}74.21 \pm 4.43^{\#} \\
(70.39-78.19)\end{array}$ \\
\hline & IA & 140 & $\begin{array}{c}71.08 \pm 5.36 \\
(66.87-76.18)\end{array}$ & $\begin{array}{l}89.62 \pm 4.45^{\star, \#} \\
(84.27-93.25)\end{array}$ & $\begin{array}{l}83.12 \pm 4.45^{*, \#} \\
(78.28-87.43)\end{array}$ & $\begin{array}{l}80.72 \pm 4.53^{*, \#} \\
(77.34-85.02)\end{array}$ \\
\hline
\end{tabular}

${ }^{\star} P<0.05$ vs. group $A,{ }^{*} p<0.05$ vs. before anesthesia.

Table VI. Adverse reactions

\begin{tabular}{|lccccc|}
\hline Group & $N$ & Irritability & Excessive sedation & Nausea & Total incidence rate (\%) \\
\hline TIVA & 140 & 5 & 11 & 0 & 11.43 \\
\hline IA & 140 & 20 & 0 & 15 & 25.00 \\
\hline
\end{tabular}


enhanced in the body, and an excessive stress response will aggravate the injury, influencing the immune function to a certain extent $[9,10]$. The perioperative stress response is mainly caused by anesthesia or surgical trauma $[11,12]$. Since the surgical method for the patient is determined, choosing an appropriate anesthetic method is of great importance to reduce the perioperative stress response and immune injury in the body. Therefore, in this study, patients undergoing laparoscopic herniorrhaphy in our hospital were prospectively selected, and the influences of different anesthetic methods on the immune function of patients were analyzed. Moreover, changes in immune function of patients were judged by detecting the levels of immune cells and inflammatory factors.

T lymphocytes are the major cells involved in cellular immunity. $\mathrm{CD}^{+} \mathrm{T}$ cells are the total $\mathrm{T}$ cells, and $\mathrm{CD}^{+} \mathrm{T}$ cells can promote $\mathrm{T}$ lymphocyte maturation and stimulate B lymphocytes to produce antibodies, while $C D 8^{+} T$ cells mainly kill target cells. Hence, the levels of these cells can represent the immune state of the body to a certain extent, and determination of these cells can reflect the immune function of patients [13]. In this study, by detecting the levels of $T$ lymphocyte subsets, it was found that $\mathrm{CD}^{+}$and $\mathrm{CD} 4^{+} \mathrm{T}$ cells and $\mathrm{CD} 4^{+} / \mathrm{CD} 8^{+}$cells were all significantly decreased at the end of the operation and at 1 day after the operation in contrast with those before anesthesia $(p<0.05)$. Moreover, at 3 days after the operation, $\mathrm{CD}^{+}$and $\mathrm{CD}^{+} \mathrm{T}$ cells were also significantly reduced compared with those before anesthesia $(p<0.05)$. However, the level of CD8 ${ }^{+} T$ cells at each detection time was not significantly different from that before anesthesia in both groups $(p>0.05)$. Also, the levels of $\mathrm{CD}^{+}$and $\mathrm{CD} 4^{+} \mathrm{T}$ cells were significantly higher in the IA group than those in the TIVA group at 3 days after the operation $(p<0.05)$. These observations suggest that anesthesia can indeed cause immune injury, and the mechanism may be as follows: On the one hand, anesthesia has a certain impact on the synthesis, differentiation and transformation of DNAs of lymphocytes, which decreases the levels of $\mathrm{CD}^{+}$and $\mathrm{CD}^{+} \mathrm{T}$ cells and causes an imbalance in $\mathrm{CD}^{+} / \mathrm{CD} 8^{+}$, resulting in the destruction of the immune barrier and the decline of immune function. On the other hand, anesthesia will also inhibit immune-related cell channels and enzyme systems, thus suppressing immune function. Meanwhile, it can be seen that TIVA has relatively little impact on the immune level of patients, probably because most inhaled anesthetics, such as enflurane and nitrous oxide, inhibit immune cells $[14,15]$. The antioxidant function and myocardial protective function of propofol are also beneficial for the maintenance of immune function.

The antioxidant system can be divided into enzymatic antioxidation and non-enzymatic antioxidation, and SOD, CAT and GSH-Px in the enzymatic antioxidant system are components of the body's physiological protection system [16]. Under external stimulation, the body will secrete more highly active molecules such as reactive nitrogen free radicals and reactive oxygen free radicals, resulting in an imbalance between antioxidant and oxidation systems, and thereby inducing tissue injury in the body. Overexpression of SOD can reduce the number of dead neurons in the hippocampal CAI region when patients have focal ischemia or global cerebral ischemia [17]. In addition, SOD can protect cortical embolism and blood-brain barrier injury after oxidative stress. The results of this study revealed that the oxidative stress indices SOD, CAT and GSH-Px declined in both groups at the end of the operation and at 1 and 3 days after the operation in contrast with those before anesthesia $(p<0.05)$, and they were significantly higher in the IA group than those in the TIVA group at each time point $(p<0.05)$. These results suggest that TIVA can effectively reduce the oxidative stress response in patients after the operation, thus decreasing the tissue injury caused by oxidative stress response.

CRP is an index to reflect the acute phase reaction after trauma, while IL-6 is a major factor that induces hepatocytes to synthesize CRP. The serum levels of IL- 6 and CRP after the operation are positively correlated with the degree of stimulation to the patients after the operation, so monitoring the dynamic changes in them can help evaluate the recovery of patients after the operation. The results of this study revealed that at the end of the operation and at 1 and 3 days after the operation, there was no obvious change in the level of TNF- $\alpha$, but the levels of IL- 6 and CRP were significantly elevated in both groups compared with those before anesthesia $(p<0.05)$. Moreover, there were significant differences in the levels of IL- 6 and CRP between the IA group and the TIVA group at the end of the operation and at 1 and 3 days after the operation $(p<0.05)$. These results 
suggest that macrophages and lymphocytes are activated in both groups after the operation. It has previously been reported that TIVA was superior to IA, probably because the protective effect of propofol on immune function suppresses the inflammatory response caused by surgical stimulation [18]. In addition, the adverse reactions were also compared between the two groups in this study. The incidence rate of adverse reactions in the IA group was significantly higher than that in the TIVA group (25.00\% vs. $11.43 \%)$. It can be seen that under the same anesthetic effect, TIVA has less impact on the immune function of patients, and the incidence rate of adverse reactions is lower.

Nevertheless, this study has some limitations. First, the dose of anesthetics was not taken into account, and indices were detected in a limited time range. Second, early complications that affected the body's systemic response were not recorded. Further in-depth studies are ongoing in our group.

\section{Conclusions}

Compared with IA, TIVA has less effect on the immune function and oxidative stress of patients undergoing laparoscopic herniorrhaphy, and controls the inflammatory response more effectively, accompanied by a lower incidence rate of adverse reactions.

\section{Conflict of interest}

The authors declare no conflict of interest.

\section{References}

1. Bloor K, Freemantle N, Khadjesari Z, Maynard A. Impact of NICE guidance on laparoscopic surgery for inguinal hernias: analysis of interrupted time series. BMJ 2003; 326: 578.

2. Mitura K, Śmietański M, Kozieł S, et al. Factors influencing inguinal hernia symptoms and preoperative evaluation of symptoms by patients: results of a prospective study including 1647 patients. Hernia 2018; 22: 585-91.

3. Öberg S, Andresen K, Klausen TW, Rosenberg J. Chronic pain after mesh versus nonmesh repair of inguinal hernias: a systematic review and a network meta-analysis of randomized controlled trials. Surgery 2018; 163: 1151-9.

4. Zenitani M, Saka R, Sasaki T, et al. Safety and efficacy of laparoscopic percutaneous extraperitoneal closure for inguinal hernia in infants younger than 6 months: a comparison with conventional open repair. Asian J Endosc Surg 2019; 12: 439-45.

5. Learning Curve in Laparoscopic Inguinal Hernia Repair: Experience at a Tertiary Care Centre. Indian Journal of Surgery 2016.
6. Bansal VK, Krishna A, Misra MC, Kumar S. Learning curve in laparoscopic inguinal hernia repair: experience at a tertiary care centre. Indian J Surg 2016; 78: 197-202.

7. China Hernia Society, Chinese Hernia College of Surgeons. Guidelines for diagnosis and treatment on the adult groin hernia (2018 edition). Zhonghua Wai Ke Za Zhi 2018; 56: 495-8.

8. Trachsel D, Svendsen J, Erb TO, Von Ungern-Sternberg BS. Effects of anaesthesia on paediatric lung function. Br J Anaesth 2016; 117: 151-63.

9. Piskin O, Altinsoy B, Çimencan M, et al. The effect of bariatric anaesthesia on postoperative pulmonary functions. J Pak Med Assoc 2017; 67: 561-7.

10. Białecki JT, Myszka W, Wysocka E, et al. A comparison of the oxidative stress response in single-incision laparoscopic versus multi-trocar laparoscopic totally extraperitoneal inguinal hernia repair. Videosurg Miniinv 2020; 15: 567-73.

11. Ramos R, Girard P, Masuet C, et al. Mediastinal lymph node dissection in early-stage non-small cell lung cancer: totally thoracoscopic vs thoracotomy. Eur J Cardiothorac Surg 2012; 41 : 1342-8.

12. Wang W, Yin W, Shao W, et al. Comparative study of systematic thoracoscopic lymphadenectomy and conventional thoracotomy in resectable non-small cell lung cancer. J Thorac Dis 2014; 6: 45-51.

13. Burfeind Jr WR, Jaik NP, Villamizar N, et al. A cost-minimisation analysis of lobectomy: thoracoscopic versus posterolateral thoracotomy. Eur J Cardiothorac Surg 2010; 37: 827-32.

14. McIntosh KR. Evaluation of cellular and humoral immune responses to allogeneic adipose-derived stem/stromal cells. In: Adipose-Derived Stem Cells. Humana Press, Totowa, NJ 2011; 133-50.

15. Ma C, Kesarwala AH, Eggert T, et al. NAFLD causes selective CD4+ T lymphocyte loss and promotes hepatocarcinogenesis. Nature 2016; 531: 253-7.

16. Oztürk-Urek R, Bozkaya LA, Tarhan L. The effects of some antioxidant vitamin-and trace element-supplemented diets on activities of SOD, CAT, GSH-Px and LPO levels in chicken tissues. Cell Biochem Funct 2001; 19: 125-32.

17. Younus $H$. Therapeutic potentials of superoxide dismutase. Int J Health Sci 2018; 12: 88-93.

18. Jin Z, Li R, Liu J, Lin J. Long-term prognosis after cancer surgery with inhalational anesthesia and total intravenous anesthesia: a systematic review and meta-analysis. Int J Physiol Pathophysiol Pharmacol 2019; 11: 83-94.

Received: 2.03.2021, accepted: 23.03.2021. 